\title{
Endoscopic treatment of prepatellar bursitis
}

\author{
Yu-Chih Huang • Wen-Lin Yeh
}

Received: 4 January 2010 /Revised: 19 April 2010 / Accepted: 19 April 2010 /Published online: 4 June 2010

(C) The Author(s) 2010. This article is published with open access at Springerlink.com

\begin{abstract}
Operative treatment of prepatellar bursitis is indicated in intractable bursitis. The most common complication of surgical treatment for prepatellar bursitis is skin problems. For traumatic prepatellar bursitis, we propose a protocol of outpatient endoscopic surgery under local anaesthesia. From September 1996 to February 2001, 60 cases of failed nonoperative treatment for prepatellar bursitis were included. The average age was $33.5 \pm 11.1$ years (range 21-55). The average operation duration was 18 minutes. Two to three mini-arthroscopic portals were used in our series. No sutures or a simple suture was needed for the portals after operation. After follow-up for an average of 36.3 months, all patients are were symptom-free and had regained knee function. None of the population had local tenderness or hypo-aesthesia around their wound. Their radiographic and sonographic examinations showed no recurrence of bursitis. Outpatient arthroscopic bursectomy under local anaesthesia is an effective procedure for the treatment of post-traumatic prepatellar bursitis after failed conservative treatments. Both the cosmetic results and functional results were satisfactory.
\end{abstract}

\section{Introduction}

The prepatellar bursa is located subcutaneously and is present in most people. Various aetiologies have been proposed as the cause of prepatellar bursitis [1-9]. Chronic inflammation of prepatellar bursa after repetitive minor

Y.-C. Huang · W.-L. Yeh $(\bowtie)$

Department of Orthopedic Surgery,

Chang Gung Memorial Hospital,

LinKou, Fu-Hsin 5, Kwei-Shan,

Tao-Yuan, Taiwan, Republic of China

e-mail: Yeh.Wen-Lin@msa.hinet.net trauma, so called "housemaid's knee", can be seen in those whose occupations require a kneeling posture, such as carpet layers and housemaids. Acute trauma, such as a fall directly onto the patella, may cause patellar bursitis [6]. The other causes of prepatellar bursitis include infection or lowgrade inflammatory conditions, such as gout, syphilis, tuberculosis, or rheumatoid arthritis. Treatment for prepatellar bursitis is determined primarily by the cause of bursitis and secondarily by the pathological change in the bursa [3]. Surgery is not required in most instances; but, if needed, the surgical procedures involved are (1) aspiration and irrigation with an appropriate drug [10], (2) incision and drainage in cases of acute suppurative bursitis [11, 12], and (3) excision of chronically infected and thickened bursa $[3,7-9,11-16]$. For the last category of patients, we usually make a transverse incision at the anterior knee region under regional or general anaesthesia, which entails admission to hospital and leaves poor cosmetic results. The resultant skin problems cause suffering in some cases. In this study, we propose an out-patient arthroscopic method under local anaesthesia aiming at a better cosmetic appearance and cost savings for the treatment of chronic prepatellar bursitis. We presume that the results of the endoscopic approach would be better than those obtained with the conventional technique.

\section{Materials and methods}

From September 1996 to February 2001, 60 patients (31 male and 29 female) with prepatellar bursitis who had failed to respond to non-operative treatments were included in this prospective study. Patients who presented with acute suppurative infection, systemic diseases (such as rheumatoid arthritis, gout, tuberculosis, diabetic mellitus, bleeding 
tendencies, etc.), alcoholism, or steroid therapy were excluded from our series. Only those who had repeated minor injuries or an apparent trauma of the ipsilateral knee were included. The average age was $33.5 \pm 11.1$ years (range 21-55). Twenty-seven patients (45\%) had a history of repeated minor knee injuries without apparent underlying cause. There were housewives, farmers, construction workers, fishermen, electronic technicians, athletes, engineers, and teachers. The repeated minor injuries were due to occupations or lifestyles in which they were frequently required to kneel. The other cases had a clear history of trauma. The mechanisms were motorcycle accidents and histories of falling down while running in the office, during matches, or while jogging, and after a contusion caused by contact with a hard object. All these patients had histories of undergoing aspiration of fluid or injection of steroid into prepatellar bursa cavities at our hospital or the other clinics. The diagnosis was based mainly on clinical examination, history, and sonographic examination. Radiographic examination included plain films of an anteroposterior and a lateral view of the knee. MRI (magnetic resonance image) or CT (computerised tomography) was not used routinely. Aspiration was performed to rule out active infection and all samples were cultured. If the culture was positive for bacterial growth, the patients were excluded from the study. Once the patients proved unresponsive to aspiration, endoscopy was immediately arranged.

\section{Operative technique}

Patients were placed in a supine position. The tourniquet was routinely applied on the thigh and was not inflated until necessary. The skin was prepared in an aseptic manner over the whole lower extremity. A $1 \%$ xylocaine with a 1:100,000 epinephrine solution was infiltrated at the portal site and then into the cavity. A $2.7-\mathrm{mm}$ diameter, 30-degree endoscope was used for monitoring. A $2.7-\mathrm{mm}$ motorised shaver was used to remove thickened synovium. Two or three $2 \mathrm{~mm}$ portals were made (Fig. 1). An anterior medial portal and an anterior lateral portal were routinely used; and, if necessary, a superior lateral portal was also made. A no. 11 scalpel was used to create a $2-\mathrm{mm}$ incision for each portal. Then the trochar was inserted into the cavity and the instruments followed. The bursa cavity and synovial thickening were directly visualised by endoscopy. The motorised shaver was inserted through the other portal, and total synovectomy (including the bursa) was performed until all the pathological lesions were removed (Fig. 2). After all the procedures were completed, the portals were closed with adhesive tape without any suture. The anterior knee area was dressed with loosely unfolded gauze and padding and bandage applied.

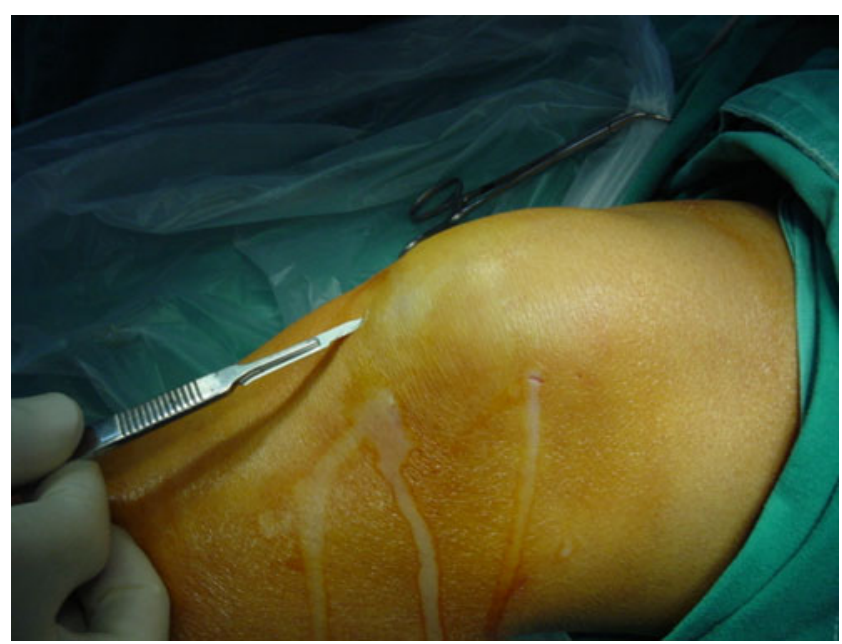

Fig. 1 The wounds were minimal as compared to a no. 15 scalpel

\section{Postoperative care}

No hospitalisation was needed. Patients went home immediately after surgery. No antibiotic was needed either before or after operation. Nonsteroidal anti-inflammatory drugs (NSAID) were given and ice packing was applied for the first 48 hours. No vigorous activity was allowed in the first two weeks. The wounds were not inspected until 48 hours later. An elastic compression bandage was applied to the knee in the first week. Patients returned to normal activities from the third postoperative week.

\section{Follow-up}

We evaluated the patients by recording swelling, pain, activities, and range of motion of their knee every month. Radiographic and sonographic examinations were made at the first and third months and every three months after operation.

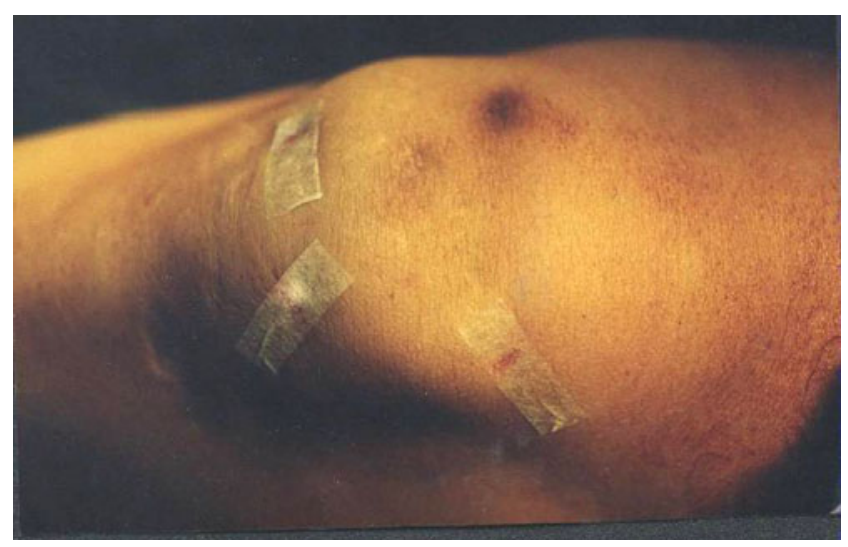

Fig. 2 Adhesive tape is usually enough to close the wounds 


\section{Results}

The average time for follow-up was $45.3 \pm 14.9$ months (range 29-87). Preoperatively, our patients received a local steroid injection an average of $3.2 \pm 2.3$ times (range 1-8) and underwent aspiration an average of $4.1 \pm 2.9$ times (range 1-12). The radiographical examination was normal in all cases. In the endoscopic operations, two portals were made for only $24(40 \%)$ patients and three portals for the other $36(60 \%)$. All cases had endoscopic findings of synovial thickening and local inflammatory vascular patches throughout the cavity (both anterior and posterior wall) (Fig. 3). No loose bodies or bony fragments were found. None of the surgical cultures produced growth in either aerobic or anaerobic groups. Average operation time was 18 \pm 5.6 minutes (range 10-25). After arthroscopic surgery, mild pain was present in $12(20 \%)$, cases but could be controlled easily with oral analgesics. In our follow-up, $80 \%$ of the patients were completely pain-free. All patients returned to their pre-injury work and activities. There was no infection nor dehiscence of the surgical wound. No limitation of their activities was noted. No tenderness or hypoethesia of the surgical scar was noted. Knee range of motion (ROM) was full in every case. Only three patients needed repeated aspiration on the fifth to tenth days after operation due to vigorous ambulation and activities against our advice. No more recurrent swelling was found. In the final radiographic examination, no heterotopic bone formation or patella infera could be perceived. The sonographic examination showed no recurrence of accumulated fluid in any of the 60 cases. No infection, wound disruption, or any other iatrogenic problems developed. No compartment syndrome occurred.

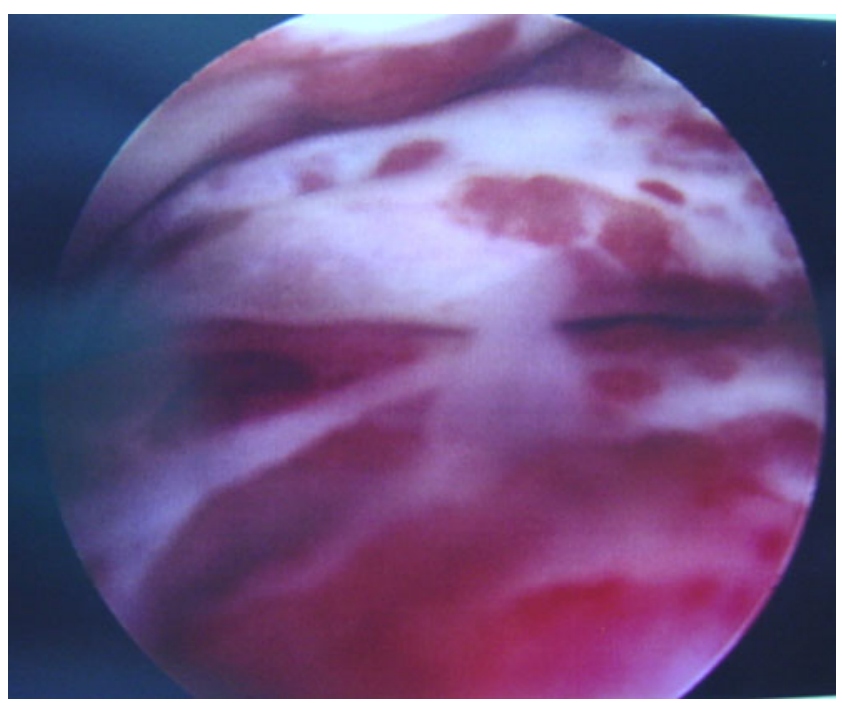

Fig. 3 The hypervascularised synovium can be identified and removed under endoscopic monitoring. The procedures were precise and the risk of skin damage can be minimised

\section{Discussion}

Prepatellar bursitis may be induced by many things, e.g. infections, tophi, rheumatoid arthritis, tuberculosis, trauma, minor injuries, or even in polio cases $[1-9,17]$. Nonoperative treatment is the mainstay for prepatellar bursitis [3]. This includes initial aspiration, applying compressive dressings, such as an Ace bandage, and administering nonsteroidal anti-inflammatory agents for ten to 14 days [18]. Due to the fact that repeated bursal aspiration is generally of little value [18], surgical excision is indicated for stubborn cases. The literature reveals that the most common complication of surgical treatment is wound problems $[3,11-15,19,20]$. Poor healing of the incision, local tenderness, or decreased sensation of scar are not rare $[2,17]$. Quayle and Robinson [11] described excision of only the posterior half of the prepatellar bursa, which is supposed to help in preventing damage to the overlying skin. Endoscopic examination of extra-articular cavities, clefts, and recesses is routinely performed today $[3,11-16$, 19-23]. Witonski [16] suggested an arthroscopic resection of prepatellar bursitis of traumatic origin with good cosmetic results and economic advantages (reduced costs, time-saving and shortened hospital stays). Kerr et al. [22] reported that arthroscopic bursectomy could prevent wound problems [11-16]; however, their patients needed hospitalisation and general anaesthesia.

In our series, we treated patients on an outpatient basis. Only local anaesthesia was infiltrated into the bursal cavity and incision sites. Since endoscopic portals were very small, no suture was needed. If tension is present after operation, a simple suture can be applied without difficulty. The operation time is short (average time 18 minutes). In terms of reducing medical costs, the economic advantages are remarkable. Our results showed these endoscopic procedures caused no infection, no limitation of range of motion, no need for lifestyle change and no skin problems. The sonographic and radiographic examinations showed no recurrence of fluid accumulation. However, we did not include infectious bursitis and systemic diseases accompanied by bursitis in our series. Gomez-Rodriguez et al. [8] stated that $95 \%$ of septic bursitis could be treated well without open surgical drainage. Pien et al. [17] described 47 episodes of septic bursitis in a private community medical practice, and the majority of patients were able to be treated as outpatients with oral antibiotics. Both authors considered surgical procedures were unnecessary in septic bursitis. On the contrary, Knight et al. [10] suggested treatment of septic olecranon and prepatellar bursitis with percutaneous placement of a suction-irrigation system with subsequent antibiotic treatment for 19 days. The role of endoscopic surgery in treatment for septic prepatellar bursitis is still unclear, and further investigation is required. 


\section{Conclusion}

For the treatment of stubborn post-traumatic prepatellar bursitis, outpatient endoscopic bursectomy under local anaesthesia is a time-saving and cost-effective procedure. Both cosmetic and functional results are satisfactory.

Open Access This article is distributed under the terms of the Creative Commons Attribution Noncommercial License which permits any noncommercial use, distribution, and reproduction in any medium, provided the original author(s) and source are credited.

\section{References}

1. Bhat AK, Bhaskaranand K (2001) Massive prepatellar bursitis in post-polio residual paralysis: a case report. J Orthop Surg (Hong Kong) 9(1):71-73

2. Butcher JD, Salzman KL, Lillegard WA (1996) Lower extremity bursitis. Am Fam Physician 53(7):2317-2324

3. Crenshaw AH (1992) Campbells's operative orthopaedics. Mosby, St Louis

4. Davis JM, Broughton SJ (1996) Prepatellar bursitis caused by Brucella abortus. Med J Aust 165(8):460

5. Dawn B, Williams JK, Walker SE (1997) Prepatellar bursitis: a unique presentation of tophaceous gout in an normouricemic patient. J Rheumatol 24(5):976-978

6. Donahue F, Turkel D, Mnaymneh W, Ghandur-Mnaymneh L (1996) Hemorragic prepatellar bursitis. Skeletal Radiol 25 (3):298-301

7. Garcia-Porrua C, Gonzalez-Gay MA, Ibanez D, Garcia-Pais MJ (1999) The clinical spectrum of severe septic bursitis in northwestern Spain: a 10 year study. J Rheumatol 26(3):663-667
8. Gomez-Rodriguez N, Mendez-Garcia MJ, Ferreiro-Seoane JL, Ibanez-Ruan J, Penelas-Cortes Bellas Y (1997) Infectious bursitis: study of 40 cases in the prepatellar and olecranon regions. Enferm Infecc Microbiol Clín 15(5):237-242

9. Papadakis KA, Vartivarian SE, Vassilaki ME, Anaissie EJ (1996) Septic prepatellar bursitis caused by Stenotrophomonas (Xanthomonas) maltophilia. Clin Infect Dis 22(2):388-389

10. Knight JM, Thomas JC, Maurer RC (1986) Treatment of septic olecranon and prepatellar bursitis with percutaneous placement of a suction-irrigation system. A report of 12 cases. Clin Orthop 206:90-93

11. Quayle JB, Robinson MP (1978) A useful procedures in the treatment of chronic olecranon bursitis. Injury 9:249

12. Quayle JB, Robinson MP (1976) An operation for chronic prepatellar bursitis. J Bone Joint Surg (Br) 58:504

13. Nussbaumer P, Candrian C, Hollinger A (2001) Endoscopic bursa shaving in acute bursitis. Swiss Surg 7(3):121-125

14. Steinacker T, Verdonck A (1998) Endoscopic therapy of prepatellar bursitis. Sportverletz Sportschaden 12(4):162-164

15. Stell IM (2000) Management of acute bursitis outcome study of a structured approach. J R Soc Med 92(10):516-521

16. Witonski D (1997) Arthroscopic resection of bursitis changes in the prepatellar bursae - preliminary report. Ortopedia Polska 62 (1):63-65

17. Pien FD, Ching D, Kim E (1991) Septic bursitis: experience in a community practice. Orthopaedics 14(9):981-984

18. McAfee JH, Smith DL (1988) Olecranon and prepatellar bursitisdiagnosis and treatment. West J Med 149:607-610

19. Freys SM (1997) Olecranon and pre-patellar bursitis. Langenbecks Arch Chir Suppl Kongressbd 114:493-496

20. Ogilvie-Harris DJ, Gilbart M (2000) Endoscopic bursal resection: the olecranon bursa and prepatellar bursa. Arthroscopy 16(3):249-253

21. Kaalund S, Breddam M, Kristensen G (1998) Endoscopic resection of the septic prepatellar bursa. Arthroscopy 14(7):757-758

22. Kerr DR (1993) Prepatellar and olecranon arthroscopic bursectomy. Clin Sports Med 12(1):137-142

23. Klein W (1996) Endoscopy of the deep infrapatellar bursae. Arthroscopy 12(1):127-131 